\title{
Benzotriazole Complexes with Amines and Phenols: Cooperativity Mediated by Induction Effects in the Crystal State
}

\author{
Cristiano Zonta, ${ }^{\dagger,{ }^{*}}$ Marco Crisma, ${ }^{\ddagger}$ Ottorino De Lucchi, ${ }^{\dagger}$ Riccardo Motterle, ${ }^{\neq}$and Siro Serafini ${ }^{\ddagger}$ \\ Istituto di Chimica Biomolecolare, CNR, Via Marzolo 1, I-35131 Padova, Italy. Dipartimento di Chimica \\ Università Ca' Foscari di Venezia, Dorsoduro 2137, I-30123 Venezia, Italy. F.I.S. Fabbrica Italiana \\ Sintetici, Viale Milano 26, I-36041 Alte di Montecchio Maggiore Vicenza, Italy.
}

\section{SUPPORTING INFORMATION}

\section{X-ray-diffraction:}

Diffraction data were collected on a Philips PW1100 diffractometer by using graphitemonochromated CuK $\alpha$ radiation $(\lambda=1.54178 \AA)$ in the $\theta-2 \theta$ scan mode up to $\theta=60^{\circ}$. The crystals were mounted on the tip of a glass fiber and coated with epoxy to prevent possible damage by air moisture. Three standard reflections, monitored at regular intervals, did not show significant

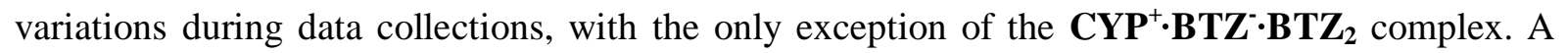
$20 \%$ linear intesity decay was observed for the latter complex. Intensities were rescaled accordingly. Unit cell parameters were obtained by least-squares refinement of the angular settings of 48 accurately centred reflections in the $12 \div 20^{\circ} \theta$ range. Intensities were corrected for Lorentz and polarization effects, not for absorption. Crystallographic data are given in Table S1. All structures were solved by direct methods with the SIR $2002^{[\mathrm{S} 1]}$ program. Refinements were carried out on $F^{2}$ by full-matrix least-squares procedures, using all data, by application of the SHELXL $97^{[\mathrm{S} 2]}$ program, with all non-hydrogen atoms anisotropic. Hydrogen atoms bound to carbons were calculated at idealized positions and refined as riding. The positions of all $\mathrm{H}$ atoms bound to nitrogen and oxygen atoms were recovered from difference Fourier maps. In the structures of $\mathbf{D C H}^{+} \cdot \mathbf{B T Z} \cdot \mathbf{B T Z}$ and $\mathbf{C Y P} \cdot \mathbf{B T Z} \cdot \mathbf{B T Z} \mathbf{T}_{2}$ the $\mathrm{H}$ atoms on heteroatoms were then refined as riding. For $\mathbf{D I P}^{+} \cdot \mathbf{B T Z}^{-}$and $\mathbf{D C H}^{+} \cdot \mathbf{B T Z} \cdot \mathbf{P h O H}$ the coordinates of $\mathrm{H}$ atoms on nitrogens were allowed to refine, while their isotropic displacement parameters were set at 1.2 times the $U_{\text {eq }}$ of the parent atom. The hydroxyl $\mathrm{H}$ atom of phenol in $\mathbf{D C H}^{+} \cdot \mathbf{B T Z} \cdot \mathbf{P h O H}$ was refined as riding.

CCDC $280181 \div 280184$ contain the supplementary crystallographic data for this paper. These data can be obtained free of charge from the Cambridge Crystallographic Data Centre via www.ccdc.cam.ac.uk/data_request/cif. 
Table S1. Crystallographic data and structure refinements for the four structures solved in this work

\begin{tabular}{|c|c|c|c|c|}
\hline Parameter & $\mathrm{DIP}^{+}: \mathrm{BTZ}^{-}(\mathbf{1})$ & $\begin{array}{l}\mathrm{DCH}^{+}: \mathrm{BTZ}^{-}: \mathrm{BTZ} \\
(\mathbf{2})\end{array}$ & $\begin{array}{l}\mathrm{DCH}^{+}: \mathrm{BTZ}^{-}: \mathrm{PhOH} \\
\text { (3) }\end{array}$ & $\begin{array}{l}\mathrm{CYP}^{+}: \mathrm{BTZ}^{-}: \mathrm{BTZ}_{2} \\
\mathbf{( 4 )}\end{array}$ \\
\hline formula & $\mathrm{C}_{12} \mathrm{H}_{20} \mathrm{~N}_{4}$ & $\mathrm{C}_{24} \mathrm{H}_{33} \mathrm{~N}_{7}$ & $\mathrm{C}_{24} \mathrm{H}_{34} \mathrm{~N}_{4} \mathrm{O}$ & $\mathrm{C}_{21} \mathrm{H}_{22} \mathrm{~N}_{10}$ \\
\hline $\mathrm{Mr}$ & 220.32 & 419.57 & 394.55 & 414.49 \\
\hline $\mathrm{T}[\mathrm{K}]$ & 293(2) & 293(2) & 293(2) & 293(2) \\
\hline$\lambda[\AA]$ & 1.54178 & 1.54178 & 1.54178 & 1.54178 \\
\hline crystal system & orthorhombic & monoclinic & monoclinic & orthorhombic \\
\hline space group & Ibca & $\mathrm{P} 2{ }_{1} / \mathrm{n}$ & $\mathrm{C} 2 / \mathrm{c}$ & Pbca \\
\hline$a[\AA]$ & $11.277(2)$ & $9.087(2)$ & $14.607(3)$ & $8.717(2)$ \\
\hline$b[\AA]$ & $11.841(2)$ & $21.565(3)$ & $17.531(3)$ & $21.365(3)$ \\
\hline$c[\AA]$ & $19.771(3)$ & $12.400(2)$ & $18.792(3)$ & $23.390(3)$ \\
\hline$\beta\left[^{\circ}\right]$ & 90 & $93.37(3)$ & $103.93(6)$ & 90 \\
\hline$V\left[\AA^{3}\right]$ & $2640.0(8)$ & $2425.7(7)$ & $4670.6(15)$ & $4356.1(13)$ \\
\hline$Z$ & 8 & 4 & 8 & 8 \\
\hline$\rho_{\text {calcd }}\left[\mathrm{Mg} \mathrm{m}^{-3}\right]$ & 1.109 & 1.149 & 1.122 & 1.264 \\
\hline$\mu\left[\mathrm{mm}^{-1}\right]$ & 0.541 & 0.559 & 0.544 & 0.667 \\
\hline$F(000)$ & 960 & 904 & 1712 & 1744 \\
\hline crystal size $[\mathrm{mm}]$ & $0.55 \times 0.50 \times 0.45$ & $0.40 \times 0.35 \times 0.30$ & $0.55 \times 0.45 \times 0.40$ & $0.60 \times 0.50 \times 0.40$ \\
\hline$\theta$ range $\left[{ }^{\circ}\right]$ & $7.03-59.98$ & $4.10-60.01$ & $4.01-59.94$ & $4.14-60.04$ \\
\hline index ranges & $\begin{array}{l}0 \leq h \leq 12 \\
0 \leq k \leq 13 \\
0 \leq l \leq 22\end{array}$ & $\begin{array}{l}-10 \leq h \leq 10 \\
-1 \leq k \leq 24 \\
0 \leq l \leq 13\end{array}$ & $\begin{array}{l}-16 \leq h \leq 15 \\
0 \leq k \leq 19 \\
0 \leq l \leq 21\end{array}$ & $\begin{array}{l}0 \leq h \leq 9 \\
0 \leq k \leq 24 \\
0 \leq l \leq 26\end{array}$ \\
\hline Refl. collected & 987 & 3985 & 3593 & 3228 \\
\hline $\begin{array}{l}\text { independent } \\
\text { reflections }\end{array}$ & 987 & $3577\left[R_{\mathrm{int}}=0.022\right]$ & $3458\left[R_{\mathrm{int}}=0.036\right]$ & 3225 \\
\hline data / parameters & $987 / 80$ & $3577 / 281$ & $3458 / 258$ & $3225 / 282$ \\
\hline $\begin{array}{l}\text { Goodness of fit } \\
\left(\text { on } F^{2} \text { ) }\right.\end{array}$ & 1.105 & 1.073 & 1.092 & 1.076 \\
\hline $\begin{array}{l}\text { Final } R \text { indices } \\
{[I>2 \sigma(I)]}\end{array}$ & $\begin{array}{l}R_{1}=0.055 \\
w R_{2}=0.147\end{array}$ & $\begin{array}{l}R_{1}=0.040 \\
w R_{2}=0.116\end{array}$ & $\begin{array}{l}R_{1}=0.071 \\
w R_{2}=0.200\end{array}$ & $\begin{array}{l}R_{1}=0.051 \\
w R_{2}=0.152\end{array}$ \\
\hline $\begin{array}{l}R \text { indices } \\
\text { (all data) }\end{array}$ & $\begin{array}{l}R_{1}=0.058 \\
w R_{2}=0.149\end{array}$ & $\begin{array}{l}R_{1}=0.048 \\
w R_{2}=0.123\end{array}$ & $\begin{array}{l}R_{1}=0.081 \\
w R_{2}=0.228\end{array}$ & $\begin{array}{l}R_{1}=0.066 \\
w R_{2}=0.162\end{array}$ \\
\hline $\begin{array}{l}\text { largest diff. peak } \\
\text { / hole }\left[\mathrm{e} \AA^{-3}\right]\end{array}$ & $0.187 /-0.235$ & $0.164 /-0.163$ & $0.351 /-0.242$ & $0.194 /-0.209$ \\
\hline
\end{tabular}




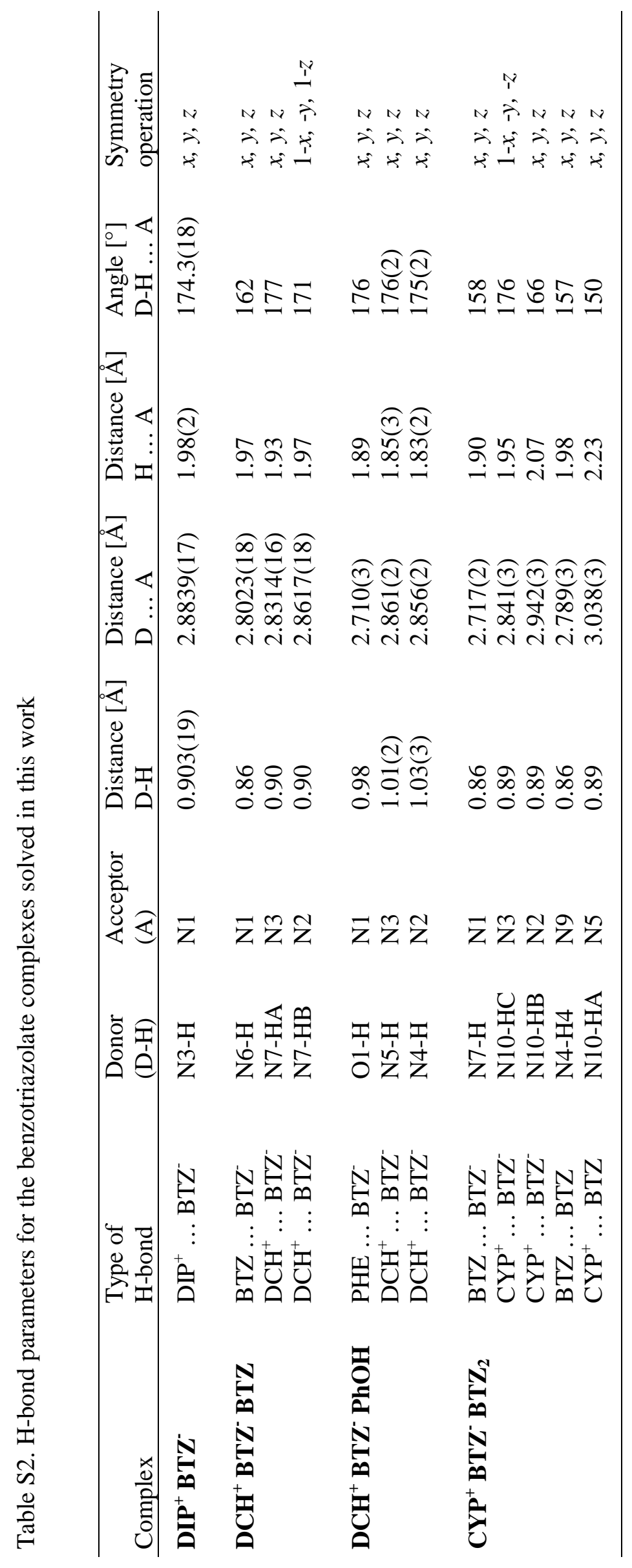




\section{Supporting references}

[S1] M. C. Burla, M. Camalli, B. Carrozzini, G. L. Cascarano, C. Giacovazzo, G. Polidori, R. Spagna, J. Appl. Crystallogr. 2003, 59, 1103.

[S2] G. M. Sheldrick, SHELXL 97. Program for the Refinement of Crystal Structures, University of Göttingen, Göttingen, Germany, 1997. 